\title{
Modelling of Diffusion in Random Packings of Core-Shell Particles
}

\section{Çekirdek-Kabuk Parçacıklarının Raslantısal Paketlemelerinde Difüzyonun Modellenmesi}

\author{
Research Article
}

\section{Emre Hatipoğlu, Harun Koku*}

Department of Chemical Engineering, Faculty of Engineering, Middle East Technical University, Ankara, Turkey.

\section{A B S TR AC T}

\begin{abstract}
Core-Shell particles are commonly used materials in chromatography. In this study, a mathematical model that mimics diffusion around Core-Shell particles was developed. A random-walk based algorithm was implemented to simulate diffusion and a Core-Shell particle geometry was computationally formed, based on simple geometric constructs and relations. Diffusion simulations were carried out on a randomly packed geometry formed from these particles. The behavior of time-dependent diffusivity data obtained from the model was found to be consistent with prior literature data from nuclear magnetic resonance experiments where transient diffusivity of a self-diffusing substance was measured in porous media.
\end{abstract}

\section{Key Words}

Core-Shell, Diffusion, Random-Walk, Geometry.

\section{öz}

\begin{abstract}
Cekirdek-Kabuk tipi parçacıklar kromatografide sıklıkla kullanılan malzemelerdir. Bu çalışmada, ÇekirdekKabuk parçacıklarının etrafında gerçekleşen difüzyonu taklit etmeye yönelik bir matematik modeli geliştirilmiştir. Difüzyonun simulasyonu için rastgele-yürüyüş temelli bir algoritma oluşturulmuş ve basit geometrik yapılar ve bağıntılardan oluşan bir Çekirdek-Kabuk geometrisi hesaplanmıştır. Bu parçacıklardan oluşan rastgele-istiflenmiş bir yapı üzerinde difüzyon simülasyonları gerçekleştirilmiştir. Model sonuçlarından elde edilen zamana bağlı difüzyon katsayısının davranışı, nükleer manyetik rezonans deneyleri vasıtasıyla gözenekli ortamlarda bir maddenin zamana bağlı öz-difüzyon katsayısının ölçüldüğü daha önceki bir çalışmanın sonuçlarıyla uyumludur.
\end{abstract}

Anahtar Kelimeler

Çekirdek-Kabuk, Difüzyon, Rastgele-Yürüyüş, Geometri.

Article History: Received: Sep 5, 2016; Revised: Nov 15, 2016; Accepted: Jan 15, 2017; Available Online: Apr 1, 2017.

DOI: $10.15671 /$ HJBC.2017.162

Correspondence to: H. Koku, Middle East Technical University, Department of Chemical Engineering, Ankara, Turkey. 


\section{INTRODUCTION}

$\mathrm{M}$ ass transfer in porous environments strongly affects the performances of systems widely utilized in fields such as catalysis and separation processes. The mass transfer performance of porous materials in chromatography is mostly governed by dispersion, which for larger molecules is dominated by intra-particle diffusion. To address limitations imposed by intra-particle diffusion, several solutions have been proposed and introduced. The class of materials known as Core-Shell particles (also known as pellicular or superficially-porous particles) is one of them.

Core-Shell particles were introduced by Horvath et al. [1] in their work related to fast liquid chromatography. Unlike fully-porous particles, the solid core of core-shell particles allow limited intraparticle diffusion which reduces band-broadening [2]. On the other hand, compared to non-porous particles, they provide a larger surface area for separation which eliminates the need for using smaller particles for better separation at the cost of increased back-pressure [3]. The popularity of core-shell particles increased especially after Halo particles were introduced by Advanced Material Technologies in 2006 [4]. Core-Shell particles are typically produced by coating solid silica spheres layer after layer (hence referred to as the layer-by-layer approach) to create the porous shell around them. This method affords a highly homogeneous pore size distribution and a smooth geometry [4].

The present study aims to create a mathematical model that explains mass diffusion around core-shell particles. Diffusion is often modelled by using Fick's Second Law of Diffusion because continuum solutions of Fick's Second Law are good approximations of the physical phenomena. However these solutions are hard to obtain and might even be inaccurate in complex geometries [5] such as packings of core-shell particles.

Considering problems with the continuum form of the Fick's Law applied to irregular geometries such as core-shell particles, a mathematical emulation of Brownian Motion is an alternative approach for modelling diffusion.
When a large amount of randomly moving particles are present in the system, the collective motion and final distribution of these particles are analogous to the continuum solution of Fick's Second Law of Diffusion in an empty media. This analogy can be extended to obstructed diffusion in porous environments by constructing an idealized geometry of the environment and checking for collisions between the random-walkers and the impermeable boundaries of their environment. There are studies that use such an approach to investigate dispersion in porous environments such as chromatographic monoliths [6] and packings of core-shell particles [7]. There are also numerous studies related to reconstruction and structural investigation of monolithic media $[8,9]$ and coreshell packings [10]. However, image-based, direct physical reconstructions of these geometries in a random-walk model have very high memory and computing power requirements. As an alternative, principles of analytical geometry can be used to formulate a typical core-shell particle geometry. This geometry can be expressed as a collection of tangent spheres, an idealized but easily obtainable approximation of the real structure, while still preserving important features of the real geometry (Solid core and porous shell). The resulting reconstruction of the geometry can be stored and used simply as a collection of center coordinates and radii of spheres. The significance of this approach is the simple representation of the core-shell particles, which circumvents huge memory and computational requirements for direct image-based methods.

\section{MATERIALS and METHODS}

\section{Random Walk}

As explained above, particle simulations based on random-walking tracers can be a good alternative to the continuum solutions of microscopic mass balances on systems with complex geometries. In Random-Walk models, the mass of the diffusing substance is represented by a statistically significant amount of tracer particles. The positions of these particles are tracked during the simulation of the diffusion event. Therefore, it is helpful to assemble them into an array $P_{i j}$ that holds particle tags and their coordinates. 
Here, index ' $i$ ' represents the $i^{\text {th }}$ particle and has the same number of elements as the amount of particles used, while index ' $\mathrm{j}$ ' is used to store the coordinates of the particles and has the same number elements as the number of spatial dimensions in the system. For instance, $P_{25,2}$ holds the $y$-coordinate of the $25^{\text {th }}$ particle, which diffuses in a system with two or more spatial dimensions.

The model is based on the following assumptions.

1. No adsorption of tracer particles.

2. The tracer particles do not interact with each other.

3. Free-solution diffusivity is isotropic and constant throughout the system.

4. Diffusing particles are point particles

5. Diffusion occurs in stagnant media.

6. No chemical reaction occurs, that can alter the material balance of the diffusing particles.

The relation between the time elapsed and the random-step length of these particles in a single dimension, is described by the following equation.

$$
\Delta \mathrm{x}=\mathrm{V}\left(2 \mathrm{D}_{\mathrm{AB}} \Delta \mathrm{t}\right)
$$

The equation implies that once a time step $(\Delta \mathrm{t})$ is chosen, the total number of random-steps that needs to be taken by the particles can be calculated simply by dividing the desired diffusion event duration by $\Delta \mathrm{t}$.

Back-calculation of diffusivity at any instant of the simulation is done by replacing by the $\Delta x$ mean displacement of all random-walking particles, and $\Delta t$ by the total time elapsed until that instant, and solving the equation for $D_{A B}$.

$$
D_{A B}=\left(\Delta x^{2}\right) / 2 \Delta t
$$

In three dimensions, every particle must move in an either positive or negative direction along each axis, with the sign of the displacement decided randomly at every step during the simulation. Accordingly, the step length of the particles, will be equal to the diagonal length of the cube with side lengths equal to $\Delta x$. The selection of the random direction can be made by generating a random number between 0 and 1. For example, the equation below will assign either +1 or -1 to the $\mathrm{RD}$ variable.

$\mathrm{RD}=\cos [$ floor $(2 * \mathrm{RN}) * \pi]$

In Equation 3, RD is a random real number between 0 and 1 , and the function floor (based on the Fortran definition) returns the greatest integer less than or equal to its argument.

Once the step-size is calculated and random numbers are generated for selection of random directions, the following equation is used to calculate coordinates of random-walking particles after each step.

$P_{i j}^{(t+1)}=P_{i j}{ }^{t}+(R D * \Delta x)$

\section{Impermeable Boundaries}

As has been explained previously, impermeability can be simulated by a collision-control mechanism between random-walking particles and impermeable boundaries. In a chromatography system involving core-shell particles in a random jammed packing, the creation of these impermeable boundaries involves three steps:

1. Creation of an idealized core-shell particle geometry

2. Creation of a random-jammed packing of identical spheres

3. Placement of core-shell geometries inside the

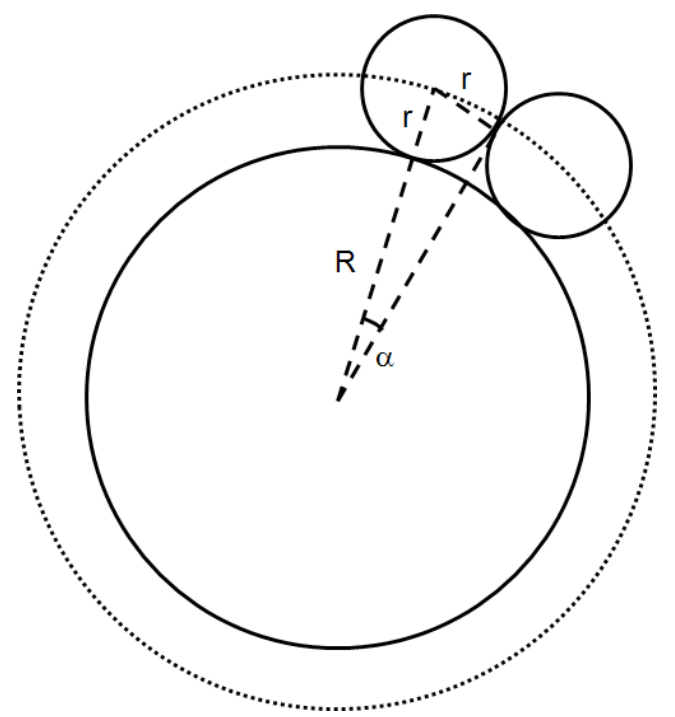

Figure 1. The core particle and two tangent shell-side particles that illustrate the first step in the generation of the geometry. The dotted guide-circle connects the centers of the shell-side spheres lined up in this manner. 
packing of identical spheres

In order to create the core-shell particle geometry, manufacturing techniques can be emulated to calculate spheres in the shell side of the particles one by one and layer by layer.

For ease of visualization, the calculation is described in terms of two dimensional references. To start, the core sphere, with radius $\mathrm{R}$, is placed, centered at the origin. The first shell-side sphere, with radius $r$, is also placed such that it lies tangent to the core sphere. Then, a hypothetical 'guide-circle' can be imagined above the coresphere (dotted circle in Figure 1), such that all shell-side spheres centered on this circle would be lying tangent to the core-sphere as well as not overlapping with the other shell-side spheres.

The maximum number of shell-side spheres (k) that can be placed around the core sphere, can be calculated by the following equation:

$$
k=f \operatorname{loor}(\pi / \alpha)
$$

Note that angle $\alpha$ (indicated in Figure 1) can be computed as the arcsine of $r /[r+R]$.

The total number of hypothetical spheres that can be placed around the core sphere in this manner can be calculated by the following

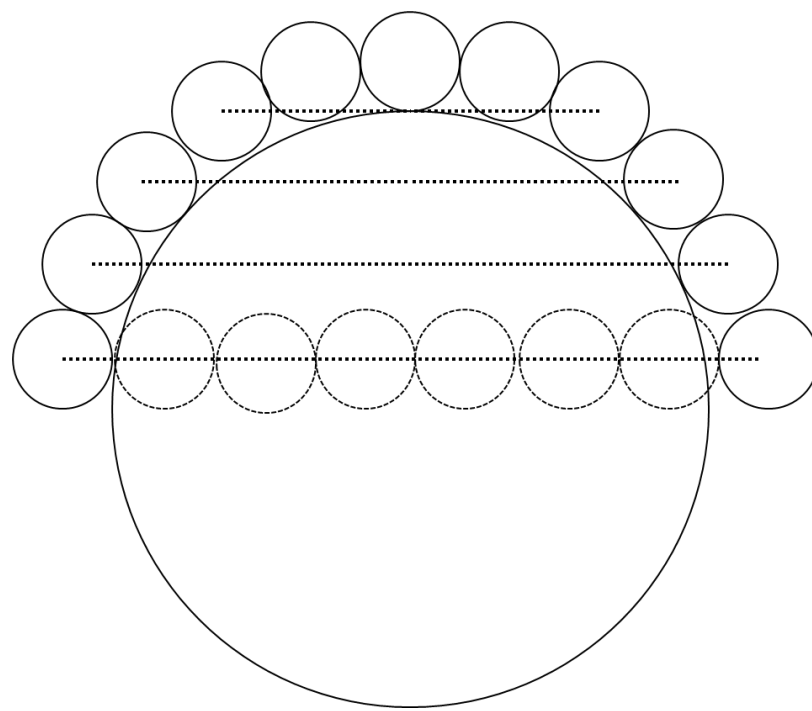

Figure 2. Extension of the guide-circles (dotted lines) to complete shell-side sphere allocation in three dimensions. The dashed circles illustrate the placement along one of the guide circles. equation.

$$
n= \begin{cases}\text { floor }(k / 2) & \text { if } k \text { odd } \\ \frac{k-2}{2} & \text { if } k \text { is even }\end{cases}
$$

Where floor is the same function in Equation 3. Using similar geometry considerations, this procedure can be extended to three dimensions. In brief, the guide-circle introduced in one dimension can be multiplied on the remaining volume of the core sphere, and the shell-side spheres can be positioned accordingly (Figure 2 ).

Algorithms and software to generate randomjammed packing of identical spheres with periodic boundaries have been made publicly available by Torquato and coworkers at the Complex Materials Theory Group [11]. Using this algorithm, it is possible to obtain the radii and central coordinates of any number of identical spheres packed randomly and tightly inside a unit cube. The packing geometry and the core-shell geometry created by the method explained in this section are suitable for any geometrical translation or scaling. Therefore the packing geometry can be shrunk or inflated from the unit cube to the same size as the coreshell geometry, then additional copies of the calculated core-shell geometry can be created inside identical spheres by simple translations.

\section{Collision Control and Periodic Boundary Conditions}

The model uses periodic boundary conditions to work around the memory and computing power requirements of creating, storing and using geometrical data that represents millions of core-shell particles packed inside a real chromatography column. A periodic randompacking of a small amount (depending on available computing power) of core-shell particles in a unit cell is created and stored. The collision control is done using the impermeable boundaries of the particles and the local coordinates of randomwalking particles inside this unit cell. This creates a virtually unbound packing of core-shell particles with the unit cell being the building block of the system.

\section{Hardware and Software Implementation of the}




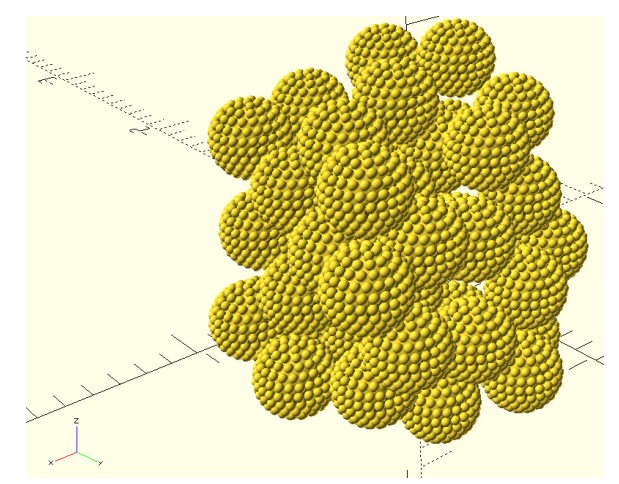

Figure 3. Three-dimensional plots of a Single Core-Shell Particle (left) and a Random-Jammed Packing of 50 Identical Core-Shell Particles inside a Unit Cell (right).

\section{Model}

For model calculations, the Fortran 95 programming language was used on a PC with $8 \mathrm{~GB} 1333 \mathrm{MHz}$ DDR3 memory and Intel Core i5 $7602.8 \mathrm{GHz}$ CPUs for computing the model. Particle-based random-walk methods require a high computing power and memory, even with the simplification of geometry by the periodic boundaries. The main factors that contribute to the CPU demand are the high resolution of the model, random-walking particle population and collision control. The wall-clock time of the computation can reach up to 250 minutes for the simulation of 60 seconds of diffusion, even with a low population and single layered coreshell geometry. To speed up the simulations, parallelization has been implemented in the code by the OpenMP. Wall-Clock time as a function of number of CPUs used follows a similar trend to Amdahl's Law due to the random number generation part in the code, which cannot be parallelized.

\section{RESULTS and DISCUSSION}

\section{Idealized Core-Shell Particle Geometries}

CAD images of idealized core-shell particle and packing geometries, drawn in OpenSCAD, are shown in the Figure 3. Visual inspection of particle geometry shows no defects or particle overlaps.

The construction method of the geometry is highly flexible such that packings with various different aspects, such as the number of shell layers, the ratio of core and shell-side particle

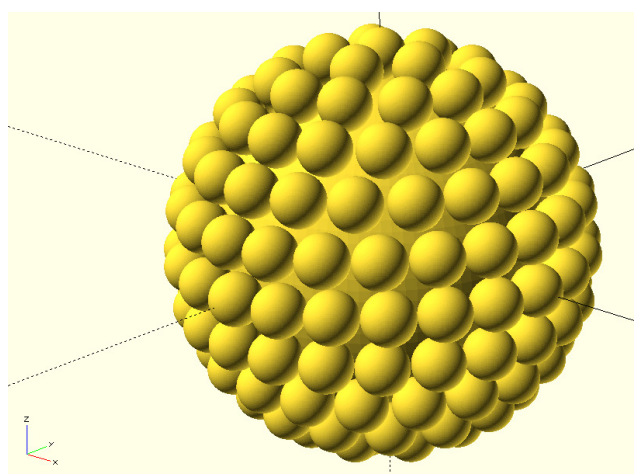

Figure 4. Time-dependent diffusivity data for 3 independent runs, normalized with respect to free-solution diffusivity $D_{0}$ in unobstructed, stagnant media. (Obtained using and).

diameters and the size of the spheres in shell layer/shell porosity, can potentially be created and used in the random-walk model to investigate their effects of on diffusion.

\section{Verification of the Random-Walk Algorithm}

Figure 4 shows changes in diffusivity of randomwalkers during the simulation in a free and stagnant environment, obtained in 3 identical runs. The probabilistic nature of the model is apparent, with the diffusivity starting with a slight fluctuation and eventually stabilizing around 110, which is the input value of diffusivity.

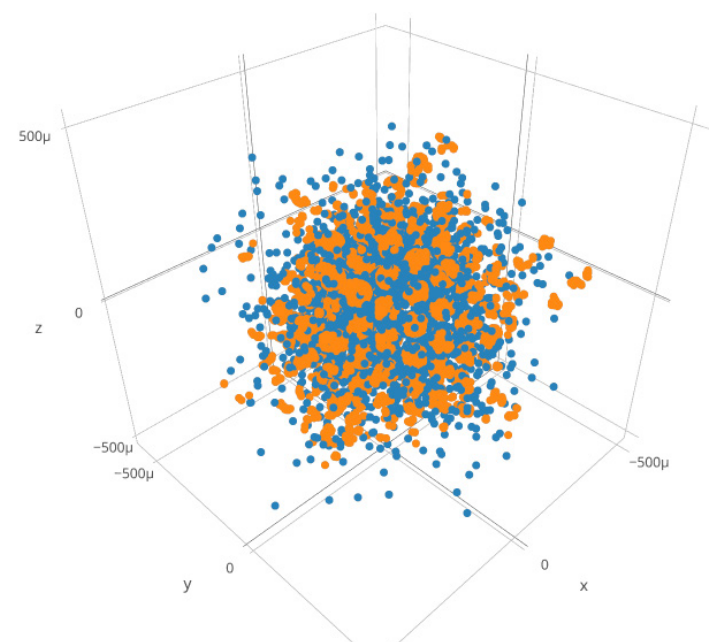

Figure 5. Final positions of the Random-Walking Particles (blue) after diffusing in an ordered packing of identical hard spheres and collision sites (orange) around impermeable hard spheres. 


\section{Verification of Collision Control \& Periodic Boundary Conditions}

The collision-control algorithm and periodic boundary conditions were tested by simulating 60 seconds of diffusion in an ordered packing of identical spheres. Simulation results were visually inspected for the verification of collision control algorithm. The scatter graph in Figure 5 shows random-walkers (tracers) in blue and collision sites between the impermeable boundaries and random-walkers in orange. Spherical patterns due to collections of collision sites confirm the validity of the collision control algorithm and the fact that there are many of these patterns shows periodic boundary condition properly creates multiplications of the unit cell used in the simulation. The collision sites seen at the outer edges of scatter graph did not experience a sufficient number of collisions to create a spherical pattern.

\section{Diffusion in Random Jammed Packing of Core- Shell Particles}

The change in the diffusivity of random-walkers over time during the simulation is shown in Figure 6. The input free-diffusivity value of $110 \mu \mathrm{m}^{2} / \mathrm{s}$ rapidly drops and gradually stabilizes around 88 $\mu \mathrm{m}^{2} / \mathrm{s}$. The features of the initial region of decline from 110 to $88 \mu \mathrm{m}^{2} / \mathrm{s}$ are dictated by the initial conditions of the simulation. All random-walkers start diffusing by point injection and they move randomly few milliseconds before starting to bump into impermeable boundaries. Therefore, their diffusivity in this time interval is close to their diffusivity in a free environment. Once randomwalkers contact core-shell particles, however, the volume available for diffusion is diminished by the presence of the solid core-shell particles in the system, resulting in a decrease in diffusivity of random-walkers compared to their diffusivity in free environment. This result is consistent with the well-known effective diffusivity definition of real substances in porous environments. Accordingly, the value $88 \mu \mathrm{m}^{2} / \mathrm{s}$ can be interpreted as the effective diffusivity of these randomwalkers in this specific geometry. This behavior is also observed in studies of nuclear magnetic resonance where time-dependent diffusion coefficients of self-diffusing substances in open

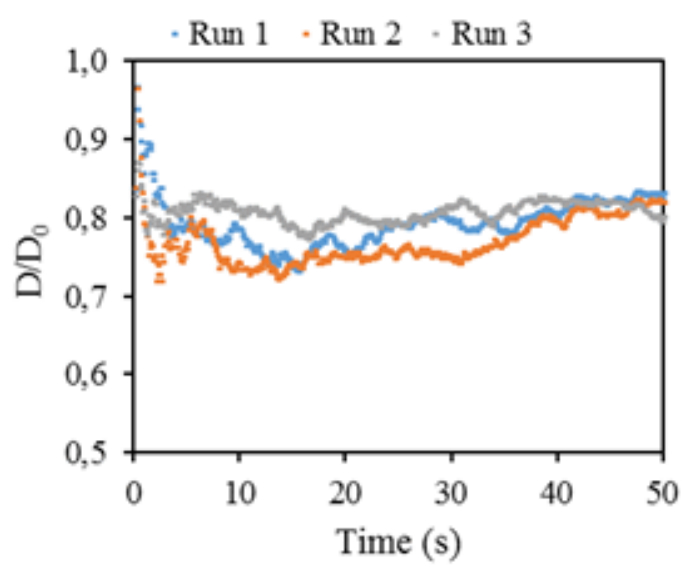

Figure 6. Time-dependent diffusivity data (normalized with respect to free-solution diffusivity, $D_{0}$ ) in a random jammed packing of idealized single layered core-shell particles for 3 independent runs (and).

porous environments are measured. Latour et al. [12] and Sen [13], have studied the time dependent diffusivity data obtained from NMR experiments to investigate the diffusion behavior in relation to porous media parameters such as tortuosity and surface-to volume ratio. Their data, obtained using tracer particles, show that normalized diffusivity starts at unity and declines to an asymptotic value essentially related to the tortuosity of the porous media $[12,13]$, very much like the results of this model shown in Figure 6.

The current model was created as the initial step of a wider research that ultimately aims to investigate dispersion in a chromatographic system involving core-shell particles. The dimensions and properties of the theoretical core-shell particles, such as the number of layers in the shell side or the diameters of shell/core spheres, should certainly have an effect on the tortuosity of the packing geometry and are worth further investigation. Additionally, it is possible to integrate fluid flow around random packings of core-shell particles, thereby coupling the flow model with the diffusion model, to investigate the behavior of more common and realistic cases in liquid chromatography.

\section{ACKNOWLEDGMENT}

This study was funded by ODTÜ BAP, as project BAP-08-112013-056. 


\section{References}

1. C.G. Horvath, B.A. Preiss, S.R. Lipsky, Fast liquid chromatography. Investigation of operating parameters and the separation of nucleotides on pellicular ion exchangers, Anal. Chem., 39 (1967) 1422 1428.

2. J.E. Macnair, K.C. Lewis, J.W. Jorgenson, UltrahighPressure Reversed-Phase Liquid Chromatography in Packed Capillary Columns, Anal. Chem., 69 (1997) 983-989.

3. K.K. Unger, R. Skudas, M.M. Schulte, Particle packed columns and monolithic columns in high-performance liquid chromatography-comparison and critical appraisal, J. Chromatogr. A, 1184 (2008) 393-415.

4. G. Guiochon, F. Gritti, Shell particles, trials, tribulations and triumphs, J. Chromatogr. A, 1218 (2011) 1915-1938.

5. F. Sattin, Fick's law and Fokker-Planck equation in inhomogeneous environments, Physics Letters A, 372 (2008) 3941-3945.

6. H. Koku, R.S. Maier, M.R. Schure, A.M. Lenhoff, Modeling of dispersion in a polymeric chromatographic monolith, J. Chromatogr. A, 1237 (2012) 55-63.

7. A. Daneyko, D. Hlushkou, V. Baranau, S. Khirevich,
A. Seidel-Morgenstern, U. Tallarek, Computational investigation of longitudinal diffusion, eddy dispersion, and trans-particle mass transfer in bulk, random packings of core-shell particles with varied shell thickness and shell diffusion coefficient, J. Chromatogr. A, 1407 (2015) 139-156.

8. E.I. Trilisky, H. Koku, K.J. Czymmek, A.M. Lenhoff, Relation of structure to performance characteristics of monolithic and perfusive stationary phases, J. Chromatogr. A, 1216 (2009) 6365-6376.

9. S. Bruns, T. Hara, B.M. Smarsly, U. Tallarek, Morphological analysis of physically reconstructed capillary hybrid silica monoliths and correlation with separation efficiency, J. Chromatogr. A, 1218 (2011) 5187-5194.

10. S. Bruns, U. Tallarek, Physical reconstruction of packed beds and their morphological analysis: Coreshell packings as an example, J. Chromatogr. A, 1218 (2011) 1849-1860.

11. M. Skoge, A. Donev, F.H. Stillinger, S. Torquato, Packing hyperspheres in high-dimensional Euclidean spaces, Physical Review E, 74 (2006) 1-11.

12. L. Latour, R. Kleinberg, P. Mitra, C. Sotak, Pore-size distributions and tortuosity in heterogeneous porous media, J. Magn. Reso., Ser. A, 112 (1995) 83-91.

13. P.N. Sen, Time-dependent diffusion coefficient as a probe of geometry, Concep. Magn. Reso., 23 (2004) $1-21$. 
